\title{
DIA/PhRMA workshop on DBS sampling in the pharmaceutical industry: methodology, implementation \& best practices
}

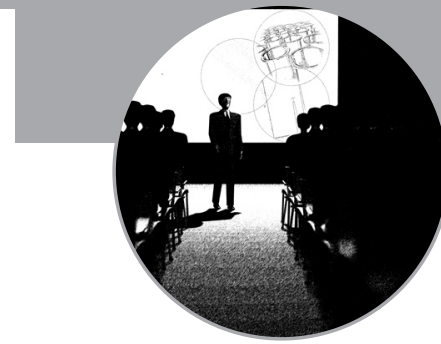

\section{The Westin Grand Hotel, Washington, DC, USA, 8 December 2009.}

Representing the first industry-led workshop on this topic, the Drug Information Association (DIA) and Pharmaceutical Research and Manufacturers of America (PhRMA) cosponsored a workshop on dried blood spots (DBS) sampling in the pharmaceutical industry. Sampling from DBS on specialty papers is a novel methodology in the area of pharmacokinetics and toxicokinetics that serves to replace conventional plasma analysis for pharmaceutical development. As the awareness around this technology has increased exponentially in recent years, this I-day symposium aimed to share current best practices for DBS use and implementation, sample collection, handling and associated bioanalysis. The event drew over 150 industry and agency participants; an impressive attendance for a relatively new technology within the industry. Overall, the day was divided into six separate technical sessions plus poster sessions, and included concurrent breakout sessions dedicated to three key areas surrounding DBS implementation: bioanalytical, toxicology/safety assessment and clinical/pharmacokinetics.

\section{Introduction to DBS sampling}

Dried blood spots (DBS) do not inherently represent a new technology; their introduction in the early 1960s is attributed to Robert Guthrie when he first applied DBS as a simple method for the detection of phenylketonuria (Følling's disease) in large populations of newborns. The method required a small volume of blood to be collected from a heel-prick, spotted onto a specialty paperbased card, dried and a small punch removed from the DBS, from which phenylalanine levels could be determined. Nearly 50 years later, in an attempt to exploit the low sampling volume potential afforded by DBS, this technology was investigated for implementation within the pharmaceutical industry. While concepts associated with DBS sampling in this industry have been presented at a variety of international and national conferences over the past 2 years, this was the first industry-led workshop, with representatives from eight pharmaceutical companies serving on the planning committee (see author list for affiliations).

\section{"While concepts associated with dried blood spot sampling in this industry have been presented at a variety of international and national conferences over the past 2 years, this was the first industry-led workshop..."}

The program chairperson Christopher Evans (GlaxoSmithKline) began the first session providing an introduction to DBS technology, where he described the potential advantages associated with DBS implementation. In a preclinical setting, the primary advantages surround the ethical benefits associated with reduced sampling volumes; while from a clinical perspective, the primary advantages revolve around simplified blood sampling (finger-prick), as well as the ability to ship and store DBS samples under ambient conditions. Evans went on to describe a 3-year 'validation' procedure of the technology within GlaxoSmithKline in which numerous in vivo studies were completed, comparing xenobiotic exposures from traditional sample matrices of plasma and wet blood with DBS. Since their implementation within GlaxoSmithKline, the technology has gone on to support numerous non-good laboratory practices (GLP) and GLP safety assessment studies, as well as a multitude of clinical studies; where at the time, approximately 35,000 DBS samples had been analyzed. Completing this introductory session, Gary Emmons (sanofi-aventis) detailed experiences validating DBS technology within his organization. He further detailed both the scientific and fiscal reasons for adoption. Using some broadly applicable assumptions for a large organization, completing numerous clinical studies with DBS, Emmons presented convincing cost savings for implementing the technology. Owing to the ability to ship and store DBS samples under ambient conditions, cost savings of greater than $€ 1$ million/year
Christopher A Evans ${ }^{\dagger 1}$, Christopher Bruce ${ }^{2}$, Gary T Emmons ${ }^{3}$, Lori Gallenberg ${ }^{4}$, Qin C Ji ${ }^{5}$, Lewis B Kinter ${ }^{6}$, Eunice Musvasva ${ }^{7}$ \& Enaksha Wickremsinhe ${ }^{8}$

'GlaxoSmithKline, 709 Swedeland Rd, Mailstop UW 2110, King of Prussia, PA 19406, USA

2Pfizer Research and Development, Sandwich, UK

${ }^{3}$ sanofi-aventis, Bridgewater, NJ, USA ${ }^{4}$ Abbott, Chicago IL, USA

${ }^{5}$ Bristol-Myers Squibb, Princeton, NJ, USA

${ }^{6}$ AstraZeneca Pharmaceuticals,

Wilmington, DE, USA

${ }^{7}$ Boehringer-Ingelheim Pharmaceuticals Inc., Ridgefield, CT, USA

${ }^{8}$ Eli Lilly and Company, Indianapolis, IN, USA

${ }^{\dagger}$ Author for correspondence:

Tel.: +l 6102707184

E-mail: christopher.2.evans@gsk.com

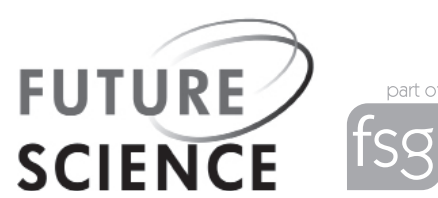


could quickly be realized. Emmons concluded with some forward-thinking ideas regarding the future of DBS to increase patient involvement in pharmacokinetic (PK) sampling in the large Phase II/III patient studies.

\section{Case studies}

The second session included a series of four case-study presentations from industry participants focusing on implementation of DBS within drug discovery, clinical development, microsampling and blood kinetics, as well as application to biopharmaceutical assets.

Utilization of DBS in exploratory drug discovery has generally been infrequent; primarily due to the large degree of method development required (e.g., paper and solvent screening). Owing to truncated timelines in drug discovery, it is not possible to screen the paper before the in vivo samples are collected; therefore, collection on inappropriate media could potentially invalidate an entire study. Starting the second session, Graeme Clark (Pfizer) described the implementation of generic methodologies to support drug discovery projects with DBS. These included the identification of a single preferred paper $\left(\mathrm{FTA}^{\circledR}\right.$ Elute), generic extraction procedures (methanol as the solvent) and the use of LC-MS/MS systems of the required sensitivity. In addition, Clark highlighted the current gaps in analytical science that need to be closed to move the technology to 'the next level' and the initiatives within Pfizer that hope to address this shortfall.

The use of DBS in clinical Phase I and II studies was presented by Gary Emmons (sanofiaventis). His organization investigated a wide diversity of approaches regarding spotting volumes, card types and punch sizes before development and validation of DBS methods starting as early as 2005 . As no issues were encountered supporting approximately 2000 samples for this antimalarial program, sanofi-aventis has continued to apply the use of DBS in additional Phase I studies in other non-antimalarial programs.

Eckhardt Schmidt (AstraZeneca) detailed studies comparing various microsampling techniques including DBS, as well as incorporating low-volume collections of liquid whole blood and plasma. AstraZeneca has evaluated DBS and has found a good correlation between traditional plasma assays, particularly in the resulting PK. Their evaluation of the technique is ongoing, with several additional studies planned in the near future.
Portfolios in the pharmaceutical industry are beginning to include an increasing number of biopharmaceuticals assets, therefore to close out the session, Jonathan Kehler (GlaxoSmithKline) shared his experiences validating a DBS method for the peptide Exendin-4, as well as a proprietary large protein $(\sim 78,000 \mathrm{amu})$, which required enzymatic digestion prior to mass analysis. Drastically different stability phenomena and recovery effects were noted during evaluation of the various card types and treatments with these analytes. Ultimately, suitable methods were developed/ validated using DMPK-C (untreated) and DMPK-A (chemically treated) cards, for the peptide and protein, respectively. In both instances the final DBS method utilized significantly less sample volume compared with the comparable plasma method and demonstrated increased analyte stability. These data represent the first demonstration of the compatibility of DBS and biopharmaceuticals.

During a brief refreshment break and poster session, attendees had the opportunity to network while viewing poster presentations. A total of 26 posters where presented by various pharmaceutical, vendor and clinical research organisations participants. Exciting poster topics included examples of bioanalytical methods, novel approaches for the use of internal standards, examination of paper homogeneity, novel uses of DBS approach to support drug discovery and the use of DBS in the area of biologics and biomarkers.

\section{Concurrent breakout sessions}

Following the poster session, sessions three and four were held as concurrent breakout sessions dedicated to three key areas surrounding DBS implementation; bioanalytical, toxicology/safety assessment and clinical/PK. Each breakout discussion group was led by at least three facilitators with intimate knowledge/experience of DBS and contained several presentations in order to facilitate discussion and questions.

\section{Bioanalysis}

This session was facilitated by Chester Bowen (GlaxoSmithKline), Qin Ji (Bristol-Myers Squibb), Laurent Vermet (sanofi-aventis) and Enaksha Wickremsinhe (Eli Lilly and Company).

The morning bioanalytical breakout session began with presentation of a 'training' video narrated by Jonathan Kehler (GlaxoSmithKline) detailing how to spot DBS cards, as well as methods by which to punch discs from the 
center of the DBS. This was followed with a presentation by Chester Bowen (GlaxoSmithKline) where he discussed the experiments necessary for validating the DBS method in a regulated bioanalytical arena. One of the key areas for discussion became the importance of demonstrating stability in whole blood (in order to account for any potential delays between sample collection and card spotting) and on-card stability. Several examples were provided in which both analytes and metabolites are more stable in DBS than corresponding 'wet' matrices, plasma or blood, an observation that could potentially be exploited for unstable analytes in the future. A final major discussion point was around assay robustness to pippetting error - will minor deviations in spot volume/size have a deleterious effect on the sample concentration? This potential issue can be assessed during method validation by evaluating a fixed size punch across different size spots of identical concentrations. Bryan Raffety (GlaxoSmithKline) and Xiao Ren (sanofi-aventis) shared data examining the distribution of drug within a DBS. Overall, the data suggest that the distribution of drug (using radiolabelled material) within a DBS may have detectable levels of variation in the distribution within the spot. However, this level of variability does not impact on bioanlaysis, particularly when spotting small volumes(smaller than $50 \mu \mathrm{l})$, and using 3-6-mm punch sizes.

The afternoon session began with a discussion of ion suppression/enhancement in the mass spectrometer by Jonathan Kehler (GlaxoSmithKline) and Qin Ji (Bristol-Myers Squibb) where data were presented showing that chemically treated DBS cards can result in substantial suppression compared with untreated cards. For this reason, and the reduced costs associated with the untreated cards, they are preferred by many of the session participants. Ji also presented a comparison of different sample clean-up procedures and introduced the term 'elution efficiency' to evaluate the effectiveness of eluting analytes from DBS cards to liquid phase during sample preparation. Laurent Vermet (sanofi-aventis) discussed the use of DBS for biologics in order to evaluate immunogenicity and $\mathrm{PK} /$ toxicokinetics measurements. He highlighted that developing the extraction process was the most challenging issue when adopting DBS for biologics, including the optimization of solubilizing the protein, selecting the buffer compositions, temperature, incubation time and volume ratio of reagent used. In addition, only nonchemically treated DBS material, such as
Whatman $903^{\circledR}$, 31ETF and ID206, should be used so protein conformational structure can be retained and the epitopes recognized by the capture or detection antibodies. Finally, Neil Spooner (GlaxoSmithKline) discussed the potential for direct analysis of DBS samples using technologies such as CAMAG's TLCMS interface, Spark Holland's DBS liquid extraction sealing surface sampling, Advion's TriVersa Nanomate and Prosolia's desorption electrospray ionization. He concluded with future challenges associated with turning these prototypes into robust instruments that can be used in a working laboratory.

In summary, the presentations and discussion from the bioanalytical breakout sessions show that DBS has numerous advantages over the traditional collection and analysis of plasma samples. Due to the fact the technique is evolving, there seems to be a need for developing or harmonizing DBS validation criteria across the industry.

\section{- Clinical/pharmacokinetics}

This session was facilitated by Kelly Connelly (GlaxoSmithKline), Gary Emmons (sanofiaventis) and Frank Hoke (GlaxoSmithKline).

In the clinical/PK breakout session, two key areas impacted by the DBS approach were explored and discussed. First, practical aspects of collecting, storing and shipping the samples were discussed after a short presentation from Rowena Sheehan (GlaxoSmithKline), who was one of the clinical staff involved in the conduct of Phase I studies. The afternoon breakout session, was a roundtable discussion led by Frank Hoke (GlaxoSmithKline) and Gary Emmons (sanofi-aventis) on the impact of using DBS as the primary matrix as opposed to plasma.

At the breakout session, a strengths, weaknesses, opportunities and threats analysis was constructed:

- Strengths included: easier sample collection, small sample volumes (e.g., pediatrics), room temperature shipment, environmentally friendly, cost savings and improved toxicokinetic assessment by serial sampling from rodents;

- Weaknesses included: possible stability/recovery issues, complex dilution process, more complicated comparison of DBS-generated PK data to that from plasma;

- Opportunities included: introduction of technique in resource-limited countries (e.g., Africa), no more blood volume restrictions for 
complicated PK studies, improved participation by patients in large studies, improvements in automation for bioanalysis;

- Threats included: concerns about paper quality, regulatory buy-in, more complicated $\mathrm{PK} /$ pharmacodynamic assessments.

\section{- Safety assessment}

This was facilitated by Loretta Gallenberg (Abbott), Eunice Musvasva (Boehringer Ingelheim) and Alan Stokes (GlaxoSmithKline).

The morning session was structured as informal discussions led by Alan Stokes, with demonstrations by Wesley Dobson (GlaxoSmithKline), on the preclinical technical aspects of sampling, spotting and handling of the cards. DBS was identified as having many key value-added traits over that of traditional study sampling practice including: ethical use of animals (replacement, refinement and reduction [3Rs]), individual animal rather than composite sampling, reduced sample storage requirements, improved sample integrity (limited potential for cross-contamination), potential for easier non-human primate sample shipment (Convention on International Trade in Endangered Species of Wild Fauna and Flora), limited safety concerns and reduced use of plastics and disposal of plastics and biological materials. Key concerns outlined were volume optimization for sample collection, loss of efficiency due to manual rather than automated procedures, application across discovery and clinical organizations and changing from plasma measurement as the currently accepted gold standard.

GlaxoSmithKline, Covance, and AstraZeneca presented materials in these sessions. GlaxoSmithKline presented supplies used in DBS and a video on the blood collection and spotting techniques. Current best practice appears to be collection of blood samples from rodent tail into coagulant-treated tubes with subsequent placement of three $15-\mu l$ samples on filter paper using a positive-displacement pipetting device.

The afternoon breakout session started with a presentation by Lewis Kinter (Astrazeneca) on the future of the DBS technique with regard to risks associated with new methodology within the preclinical sciences and potential impact toward regulatory acceptance of preclinical findings and safety assessments. Robust discussion across preclinical and GLP quality assurance scientists included concerns about acceptance within regulatory agency, quality, clinical and pharmaceutical decision makers. Key to acceptance will be understanding the GLP and good clinical practice regulatory needs, scientific rigor applied to the method and thorough knowledge of parent and metabolite measurements in relevant species. Proposals were made to openly share information across pharmaceutical companies to assure consistent practices akin to those for plasma measurements and quickly share data with regulators to gain acceptance and limit perception of risk.

Test article contamination of control samples has been detected by GlaxoSmithKline in DBS studies and was thought to be related to aerosolized test article. To control/detect this type of ex vivo contamination, GlaxoSmithKline technicians recommended conduct of spotting and drying of blood samples in a separate study room from animal dosing and blood collection, and spotting of separate 'naive blood' samples. There were discussions around submission of these data to regulatory agencies and what comments/feedback has been received. At least one investigational medicinal product dossier has been submitted containing preclinical DBS sampling, with no regulatory objection.

“...by many factors the symposium was deemed a huge success. A diverse group of scientists came together to discuss a novel and emerging topic in a nonbiased format; ideas were shared, concepts discussed and topics debated."

\section{Regulatory perspective}

Michelle Beharry, a nonclinical assessor from Medicines and Healthcare products Regulatory Agency, provided an evaluation of the DBS technique from a regulatory perspective. This agency is responsible for the regulating the safety of medicines and medical devices in the UK; therefore, in her presentation Beharry strongly encouraged implementation of DBS technology owing to ethical benefits that can be realized to both preclinical species and patients. Furthermore, she detailed how open the agency was towards discussions with sponsors and encouraged consultation with the agency during any stage of the study, particularly during the planning stages.

\section{Synopsis \& questions \& answers}

The final session was an interactive discussion and summary of the major talking points and themes from the various breakout sessions. The culmination of the various presentations 


\section{CONFERENCE Report | News \& ANALYSIS}

and discussions throughout the day identified several key areas for further investigation and understanding. These topics will no doubt be the subject of some upcoming active research and publications. A lively questions and answers session around the pros and cons of DBS implementation ensued. The symposium adjourned with a networking reception where pharmaceutical colleagues had a final opportunity to discuss and revel in the learnings from the day.

Overall, by many factors the symposium was deemed a huge success. A diverse group of scientists came together to discuss a novel and emerging topic in a nonbiased format; ideas were shared, concepts discussed and topics debated.

\section{Acknowledgements}

The planning committee wishes to acknowledge the Drug Information Association and Pharmaceutical Research and Manufacturers of America for sponsorship and planning (most specifically Rachel Minnick and Jo Ann Boileau), breakout session facilitators, as well as all of the poster presenters. Without their efforts this symposium would not have been such a successful event.

\section{Financial \& competing interests disclosure}

The authors have no relevant affliations or financial involvement with any organization or entity with a financial interest in or financial conflict with the subject matter or materials discussed in the manuscript. This includes employment, consultancies, honoraria, stock ownership or options, expert testimony, grants or patents received or pending, or royalties.

No writing assistance was utilized in the production of this manuscript. 